\title{
Références bibliographiques du dossier « Former des enseignants »
}

\section{Marion Latour}

\section{(2) OpenEdition \\ 12 Journals}

Édition électronique

URL : http://journals.openedition.org/ries/994

DOI : 10.4000/ries.994

ISSN : 2261-4265

Éditeur

Centre international d'études pédagogiques

Édition imprimée

Date de publication : 1 décembre 2010

Pagination : 151-162

ISBN : 978-2-8542-583-1

ISSN : $1254-4590$

Référence électronique

Marion Latour, « Références bibliographiques du dossier « Former des enseignants » », Revue internationale d'éducation de Sèvres [En ligne], 55 I décembre 2010, mis en ligne le 05 juillet 2011, consulté le 20 avril 2019. URL : http://journals.openedition.org/ries/994 ; DOI : 10.4000/ries.994

(ㄷ) Tous droits réservés 


\section{Références \\ bibliographiques}

\section{Marion Latour}

La formation des enseignants, tant initiale que continue, est au cœur du débat éducatif, que ce soit dans les pays développés ou dans les pays moins développés où l'on cherche à rendre l'éducation accessible à tous. En témoigne l'abondante littérature sur le sujet. La sélection bibliographique proposée organise les documents les plus pertinents selon trois axes:

- ce qui fait débat et renvoie aux enjeux de la formation à l'enseignement, dans sa dimension politique et en évoquant le rôle de la recherche en éducation ;

- le contenu et les modalités de la formation des enseignants, généralement conçue dans une continuité entre formation initiale et formation continue ; à l'exception de quelques documents spécifiques à la France, les études transnationales et comparatistes ont été privilégiées, avec une distinction entre pays de l'OCDE et pays en développement (les bibliographies des articles fournissant des données nationales sur d'autres pays);

- la notion de compétence, en cherchant à définir les compétences professionnelles en jeu, leur évaluation et le développement professionnel tout au long de la carrière des enseignants et en prise avec l'évolution du métier.

La majorité de ces publications, postérieures à 2006, sont consultables au Centre de ressources et d'ingénierie documentaires du CIEP ou accessibles en ligne.

Bibliographie arrêtée le 30 septembre 2010.

\section{LES DÉBATS ET ENJEUX DE LA FORMATION DES ENSEIGNANTS}

Commission européenne, Améliorer la qualité des études et de la formation des enseignants : Communication de la Commission au Conseil et au Parlement européen, août 2007, 17 p. téléchargées du site http://eur-lex.europa.eu, stat.

Cette communication dresse un panorama des études et de la formation des enseignants dans l'Union européenne, en rappelant tout d'abord le contexte de l'agenda de Lisbonne pour la croissance et l'emploi. Elle propose une réflexion sur les mesures que peuvent prendre les États membres et sur l'aide que l'Union peut apporter. Elle offre une vision de la profession d'enseignant en Europe qui se fonde sur des principes communs, notamment en termes de compétences nécessaires et de qualifications.

\section{CLANET JOël dir., Recherche/formation des enseignants : quelles articulations ?,} Presses universitaires de Rennes/Rennes, 2009, 184 p., bibliogr. (Paideia - Éducation Savoir Société)

L'ouvrage interroge les relations possibles entre les travaux de recherche portant sur les pratiques enseignantes et la formation professionnelle des enseignants dans les contextes français, suisse et québécois. Cette analyse s'organise en trois temps : un panorama de ce qui existe et a existé en matière de liens entre recherche et formation des enseignants; des travaux où les liens recherche/formation sont clairement explicités; enfin des travaux de recherche qui n'ont pas d'application directe mais sont destinées à nourrir la réflexion des enseignants sur leur propre pratique. 
CSEE: Comité syndical européen de l'éducation/Bruxelles, La formation des enseignants en Europe - Document politique du CSEE, CSEE : Comité syndical européen de l'éducation/Bruxelles/Belgique, 2008, 74 p. téléchargées du site http:// etuce.homestead.com/

Cet ouvrage expose la vision du Comité syndical européen de l'éducation (SCEE) en matière de formation des enseignants. Après une présentation des raisons qui ont incité le SCEE à élaborer une nouvelle politique sont abordés la formation initiale des enseignants, la situation des formateurs (des enseignants), la politique de recrutement et de maintien en service, enfin, le développement professionnel continu, puis les défis à relever dans la société actuelle qui repose sur la connaissance.

ETIENNE Richard dir., ALTET Marguerite dir., PAQUAY Léopold dir., et al., L'université peut-elle vraiment former les enseignants? Quelles tensions? Quelles modalités ? Quelles conditions?, De Boeck Université/Bruxelles, 2009, 264 p. (Perspectives en éducation et formation)

Dans la plupart des pays, la formation des enseignants s'inscrit dans un double mouvement. L'augmentation de la proportion d'expérience de terrain doit permettre une professionnalisation accrue de la formation et l'élévation du niveau universitaire est considérée comme indispensable pour assurer la maîtrise des savoirs à enseigner et pour enseigner. Cette mise en place ne va pas de soi et nécessite la réalisation de certaines conditions et transformations pour sa mise en œuvre. Les différentes contributions analysent les tensions d'une formation professionnelle à l'université à partir des situations en Belgique, au Québec et en France).

FEYFANT Annie, L'apprentissage du métier d'enseignant, INRP/Cellule de veille scientifique et technologique/Lyon, 2010, 12 p. téléchargées du site http://www. inrp.fr, bibliogr.

Le dossier est organisé en trois volets : modalités de formation, approche théorique du métier et alternance entre acquisitions de savoirs d'expérience et acquisition de savoirs pédagogiques. L'auteure analyse la situation en France et la réforme engagée en 2008 et s'appuie sur les systèmes de formation de pays européens (Belgique, Irlande, Allemagne) et nord-américains (États-Unis, Québec) pour montrer la nécessaire articulation entre pratique professionnelle et apports théoriques.

JAROUSSE Jean-Pierre coord., La scolarisation primaire universelle en Afrique : le défi de l'enseignant, Pôle de Dakar/Dakar, 2009, 197 p. téléchargées du site http://www.poledakar.org/, bibliogr.

On estime aujourd'hui qu'environ quatre enfants africains sur dix ne terminent pas l'école primaire, résultat très éloigné de l'objectif du forum de Dakar en 2000 d'assurer la scolarisation primaire universelle à tous les enfants d'Afrique. Sur ce continent, la question enseignante est l'élément central des politiques actuelles en matière de développement des systèmes éducatifs. Cette publication s'attache à montrer d'autres dimensions que la maîtrise des coûts salariaux comme la formation, la gestion ou le développement professionnel des enseignants. L'ouvrage est organisé en cinq chapitres : présentation du contexte et des contraintes propres aux pays africains, questions de rémunération, de statuts, de qualité et de gestion de l'école et des enseignants.

LANTHEAUME Françoise ed., Le travail enseignant : crises et recomposition du local à l'international, retour sur le métier, Recherche et formation, 2008, $n^{\circ} 57,171 p$.

Les contributions tentent de rendre compte du travail des enseignants dans sa complexité, en focalisant la problématique vers l'activité réelle et le développement à la fois personnel et professionnel. Les évolutions du travail enseignant, envisagées d'abord dans une perspective 
socio-historique interrogeant le sens du mouvement volontariste de professionnalisation, sont ensuite étudiées sous différents angles d'approches, à l'échelle internationale, nationale et locale, dans les situations les plus quotidiennes.

PERRENOUD Philippe dir., ALTET Marguerite dir., LESSARD Claude dir., Conflits de savoirs en formation des enseignants, De Boeck Université/Bruxelles, 2008, 274 p., bibliogr.

Les différents contributeurs abordent un problème central de la formation des enseignants : les rapports difficiles entre savoirs des praticiens et savoirs issus de la recherche. Leur alliance ne va pas de soi car ces savoirs diffèrent par leurs origines, leur langage et leur degré d'abstraction, leur rapport à l'action et leurs critères de validité. Ils sont portés par des acteurs différents, les enseignants et les chercheurs. Les formateurs d'enseignants sont souvent des médiateurs entre ces deux mondes. Cet ouvrage analyse les obstacles qui rendent difficiles les métissages entre savoirs. Il propose aussi des dispositifs, dans le cadre plus global des réflexions contemporaines sur l'alternance, l'articulation théorie-pratique, l'apprentissage par problèmes et le développement de compétences.

RAYOU Patrick coord., Des enseignants pour demain : dossier, Éducation et sociétés, 2009, $n^{\circ} 23$, p. 5-168

La profession enseignante connaît une crise profonde à l'échelle internationale : compressions budgétaires, influence des nouvelles technologies, marginalisation croissante d'une certaine partie de la jeunesse, crise du savoir... avec pour conséquence des difficultés de recrutement et des situations de pénurie de professeurs dans certains pays. Les différentes contributions ont été regroupées selon trois axes. Le premier est celui de l'histoire du système éducatif et des politiques scolaires qui l'ont structuré, le deuxième axe précise le rôle des acteurs, les manières nouvelles d'exercer le métier d'enseignant et leur prise en compte par l'institution. Le troisième axe s'intéresse à la dialectique entre l'individuel et le collectif dans les transformations actuelles.

SCHWILLE John, DEMBELE Martial, SCHUBERT Jane coll., Former les enseignants : politiques et pratiques, IIPE/Paris, 2007, 164 p., bibliogr. (Principes de la planification de l'éducation)

À partir de l'examen critique et sélectif de la littérature sur la formation initiale et professionnelle continue des enseignants, les auteurs traitent de questions controversées telles que la durée de la formation, ses formes possibles, les politiques et les pratiques d'une formation professionnelle initiale, interrogeant même la nécessité d'une telle formation. Puis ils abordent le type de soutien dispensé aux enseignants débutants ainsi que la formation professionnelle continue.

WENTZEL Bernard dir., MELLOUKI M'hammed dir., Recherche et formation à l'enseignement : spécificités et interdépendance, Haute École Pédagogiquel BEJUNE/KL/Suisse, 2010, 173 p.

Des chercheurs suisses, canadiens et français apportent réflexions et témoignages sur la place de la recherche dans les institutions, les programmes et les activités de formation à l'enseignement. Ils constatent l'ouverture des programmes aux connaissances issues de la recherche en éducation, la mise en œuvre de dispositifs de formation scientifique pour de futurs enseignants et la rénovation des principes pédagogiques d'une alternance entre théorie et pratique. 


\section{L'ORGANISATION \\ DE LA FORMATION INITIALE \\ ET CONTINUE}

\section{Études transnationales}

\section{Pays de l'OCDE}

BARRERE Anne réd., LEGRAND Guy réd., La formation des enseignants : des IUFM aux masters. Dossier, Recherche et formation, INRP/Paris, 2009, $n^{\circ}$ 60, p. 5-140

Ce numéro retrace l'évolution de la formation des enseignants en France et en Suisse, tout en réfléchissant aux notions d'identité professionnelle, de professionnalité et de compétence professionnelle, à la situation des enseignants débutants et à la formation des enseignants dans certaines disciplines telles que les sciences économiques et sociales ou la technologie.

Committee on the Study of Teacher Preparation Programs in the United States, National Research Council/Washington, Preparing teachers: building evidence for sound policy, National Academies Press/Washington, 2010, 234 p.

L'ouvrage s'intéresse aux candidats à l'enseignement et à la formation qu'ils reçoivent aux États-Unis, plus particulièrement en termes de contenus, de connaissances et de compétences pédagogiques visées et d'efficacité. Le besoin émerge d'un modèle pour collecter les données afin de fournir une information fiable sur la formation des enseignants à l'usage des décideurs politiques, tant à l'échelle fédérale qu'à celle des États.

GRAU Sylvie coord., ÉTIENNE Richard coord., Quelle formation pour les enseignants ?, Cahiers pédagogiques, juillet 2010, HS n 17, 492 p., bibliogr.

Ce numéro spécial dédié à la formation des enseignants débute par les questions brûlantes soulevées par le débat actuel, telles que la préparation au métier et la réforme de la mastérisation. Puis il propose des repères historiques pour comprendre l'évolution du métier et de la formation en France et des descriptions de la formation professionnelle en Belgique, en Suisse, au Québec et aux États-Unis. Les contributions suivantes abordent l'entrée dans le métier et son accompagnement, les contenus et modalités pour la formation initiale et la formation continue.

HUDSON Brian, ZGAGA Pavel, ASTRAND Bjorn, Advancing quality culture for teacher education in Europe: tensions and opportunities, 2010, 301 p. téléchargées $d u$ site http://www.pef.uni-lj.si, index thématique et d'auteurs, bibliogr.

Après deux articles introductifs traitant de l'impact du Processus de Bologne sur la formation des enseignants et la question de la qualité et du statut des formateurs d'enseignants, cinq articles analysent la formation des enseignants dans plusieurs contextes géographiques d'Europe ainsi qu'au Québec. Les contributions suivantes s'intéressent à des aspects spécifiques tels que la construction de l'identité professionnelle, les rapports qu'entretiennent les enseignants avec la recherche, la préparation des futurs enseignants et le concept de connaissances au sein de la formation des enseignants.

LARUELLE Olivier, Les politiques de formation continuée des enseignants : une approche comparative, Presses universitaires de Namur/Namur, 2009, 106 p. (Politiques)

Cet ouvrage examine les politiques de formation continue des enseignants dans différents pays européens ainsi qu'au Québec et en Corée du Sud. L'approche comparative s'appuie sur des 
indicateurs communs élaborés comme grille d'analyse de ces politiques. La deuxième étape propose une évaluation des pratiques de formation et des innovations en la matière à partir des indicateurs de la grille d'analyse.

\section{MALET Régis, La formation des enseignants comparée. Identité, apprentissage} et exercice professionnels en France et en Grande-Bretagne, Peter Lang/Berne/ Suisse, 2008, 258 p.

S'appuyant sur de nombreuses recherches empiriques menées en France et en Grande-Bretagne, cet ouvrage s'intéresse à la construction des identités professionnelles des enseignants et aux dispositifs d'apprentissage et d'exercice du métier. Il aborde notamment les processus de médiation qui entrent en jeu dans la formation des enseignants.

MALET Régis, «Former, réformer, transformer la main-d'œuvre enseignante? Politiques comparées et expériences croisées anglo-américaines ", Éducation et sociétés, 2009, $n^{\circ} 23$, p. 91-122, bibliogr.

Cet article comparatif entre la Grande-Bretagne et les États-Unis montre la redéfinition du rôle de l'État dans la gouvernance du secteur public. Alors que l'on observe un contrôle direct des établissements et des enseignants en Angleterre, la dérégulation et le contrôle indirect de la profession est de mise outre-Atlantique. L'auteur pose la question de la formation des enseignants sous l'angle des enjeux économiques et de l'obsession de la performance ; il questionne les réformes actuelles et leur impact sur la professionnalité des enseignants.

OCDE: Organisation de coopération et de développement économiques/Paris, Educating teachers for diversity: meeting the challenge, OCDE/Paris, 2010, 313 p. (Educational research and innovation)

L'ouvrage examine les concepts sous-jacents à la diversité dans différents pays de l'OCDE pour améliorer l'articulation entre la formation initiale et continue des enseignants. Plusieurs chapitres examinent la formation des enseignants et les stratégies utilisées pour préparer les praticiens à la diversité dans la classe dans des pays emblématiques tels que la Nouvelle Zélande, les Pays-Bas, l'Italie, l'Allemagne, l'Espagne, l'Irlande du Nord et les États-Unis.

\section{Pays en développement}

BRUNEFORTH Michael, GAGNON Amélie, WALLET Peter, Projections de la demande mondiale d'enseignants : atteindre l'objectif de l'enseignement primaire universel d'ici 2015, 2010, 46 p. téléchargées du site http://unesdoc. unesco.org/, annexes

Ce document présente les nouvelles projections mondiales préparées par l'Institut de statistique de l'UNESCO (ISU) afin de quantifier l'écart du nombre d'enseignants en matière d'enseignement primaire. Il approfondit aussi les concepts, les hypothèses et les techniques de calcul du modèle de projection de l'ISU et regroupe des renseignements permettant une utilisation éclairée des résultats.

Capacity trust \& Pedium, Evaluation of the Teacher Training Initiative for SubSaharan Africa (TTISSA), août 2009, 63 p. téléchargées du site http://unesdoc. unesco.org/ [consultées le 5 octobre 2009]

L'Initiative pour la formation des enseignants d'Afrique subsaharienne, TTISSA, a été initiée en janvier 2006 dans dix-sept pays de la zone pour augmenter en quantité et en qualité l'offre d'enseignants, afin d'atteindre l'objectif d'une éducation primaire universelle. Le présent rapport évalue la pertinence, l'efficacité, l'efficience et l'impact de ce dispositif. 
CROS Françoise, BERIOT Anne-Marie, Professionnaliser les nouveaux enseignants: prospectives forces et apports des séminaires conduits par le Centre international d'études pédagogiques 2006-2009 = Professional skills training for new teachers: prospects strengths and outcomes of the seminars organised by the Centre international d'études pédagogiques, CIEP/Sèvres, 2010, 23 p. + $23 \mathrm{p}$.

Fruit de la réflexion menée lors de trois colloques internationaux sur la formation des enseignants à l'heure de l'éducation pour tous. L'ouvrage propose notamment une typologie des dispositifs de professionnalisation selon les différentes situations de l'enseignant (l'enseignant sans formation initiale et l'enseignant débutant en particulier) et une série de recommandations à plusieurs niveaux : celui des décideurs et des politiques, celui des responsables régionaux, enfin celui des responsables des dispositifs et des formateurs.

CROS Françoise, FRELAT-KAHN Brigitte, Professionnaliser les enseignants sans formation initiale : des repères pour agir : actes 2-6 juin 2008 = Profesionalizar a los profesores sin formacion inicial : puntos de referencia para actuar : actas 2-6 de junio de 2008, CIEP : Centre international d'études pédagogiques/Sèvres, Agence Française de Développement/Paris, Ministère des Affaires étrangères/Paris, UNESCO/Paris, 2009, 302 p. + CD, annexes

Après un chapitre introductif sur les enjeux de la professionnalisation des enseignants sans formation initiale, des chercheurs et experts confrontent leurs approches. Le troisième chapitre expose la situation sur le terrain dans des pays d'Afrique, d'Amérique Latine, d'Asie et au Liban. Une synthèse des travaux en ateliers est proposée avant d'aborder des éléments de conclusion et les questions en suspens et de proposer des pistes pour l'action.

\section{KARSENTI Thierry dir., GARRY Raymond-Philippe dir., BENZIANE Abdelbaki} dir., Former les enseignants $d u X X I^{e}$ siècle dans toute la francophonie, AUF/ Montréal, Presse Universitaire Blaise Pascal/Université de Clermont 2/Clermont, 2008, 380 p., bibliogr.

Le Réseau international francophone des établissements de formation de formateurs, RIFEFF, a pour mission de promouvoir la coopération entre les institutions francophones ouvrant pour la formation de formateurs, en vue de contribuer à l'amélioration de la qualité de l'enseignement et à la professionnalisation des métiers de l'éducation. L'ouvrage traite de la formation des enseignants dans les pays francophones à travers quatre thématiques : le français, vecteur d'interculturalité ; un master francophone de formation des enseignants ; les TIC, outils de complémentarité pour la formation initiale et continue; la formation des maîtres et l'éducation pour tous.

International Reading Association/Newark/Etats-Unis, Status of teacher education in the Asia-Pacific region, UNESCO, 2008, 140 p. téléchargées du site http:// www.reading.org

Cette description de l'organisation de la formation des enseignants dans vingt-cinq pays de la région Asie-Pacifique permet de dégager quelques grandes tendances communes, au nombre desquelles la fragmentation des infrastructures d'enseignement, la question du déploiement des enseignants, l'absence de normes pour la qualification, la rétention des enseignants les mieux qualifiés, le rôle insuffisant des associations professionnelles, la contribution de la recherche et l'impact du post-colonialisme. 
MULKEEN Aidan, CHAPMAN David, DeJAEGHERE Joan, et al., Recruiting, retaining and retraining secondary school teachers and principals in SubSaharan Africa, World Bank/Washington/États-Unis, mars 2007, 75 p., bibliogr., stat. World Bank working paper

Cette étude, qui porte plus particulièrement sur l'Éthiopie, le Ghana, la Guinée, Madagascar, la Tanzanie et l'Ouganda, repère les bonnes pratiques pour mieux former les enseignants du secondaire, qui passent par de nouveaux modes de recrutement, le développement de la formation initiale et continue, un meilleur déploiement des enseignants, des compensations et une amélioration des conditions de travail.

MULKEEN Aidan, Teachers in Anglophone Africa: issues in teacher supply, training and management, Banque mondiale/Washington, 2010, 199 p., bibliogr., index

Ce rapport dresse un panorama de la situation des enseignants dans les pays d'Afrique anglophone. Après un rappel du contexte et des grandes tendances sont abordés l'offre d'enseignants et leur répartition géographique, la formation (initiale et continue), le développement professionnel, l'inspection et l'évaluation, la rétribution, l'absentéisme, la carrière et le financement des enseignants.

PINI Monica, GOROSTIAGA Jorge, "Teacher education and development policies: critical discourse analysis from a comparative perspective”, International review of education, juillet 2008, vol. 54, $n^{\circ} 3-4$, p. 427-443, bibliogr., annexes

En s'appuyant sur une sélection de documents récents de politique éducative au niveau national et international, cet article explore et compare les politiques de formation des enseignants dans plusieurs pays d'Amérique Latine et d'Amérique du Nord.

\section{Études sur la France}

BOISSINOT Alain, MICHEL Alain, POCHARD Marcel, et al., "Enseignant : un métier en mutation ", Administration et éducation, décembre 2008, n 120, 207 p.

En proposant une double approche, à la fois macroscopique pour le statut, les missions, le recrutement, la formation et l'évaluation des enseignants, et une vision de terrain pour l'activité dans la classe et au sein de l'établissement, ce numéro apporte analyse et témoignage sur la formation des enseignants en France.

Cahier des charges de la formation des maîtres en Institut universitaire de formation des maîtres, JO du 28 décembre 2006, arrêté du 19 décembre 2006, NOR : MENS0603181A

Outre une définition de l'organisation et des objectifs de la formation initiale des enseignants en France et des principes sur lesquels repose la formation professionnelle, l'arrêté décrit en annexe dix compétences professionnelles devant être prises en compte dans la formation de tous les enseignants. Chacune met en jeu des connaissances, des capacités et des attitudes professionnelles fondamentales.

CPU : Conférence des présidents de l'université/Paris, CDUL: Conférence des directeurs des UFR de lettres langues arts et sciences/Paris, CDIUFM : Conférence des directeurs d'IUFM/Paris, et al., États généraux de la formation des enseignants : synthèse des contributions : bilan et perspectives, septembre 2009, 44 p. téléchargées $d u$ site http://www.etatsgeneraux-formationdesenseignants.fr/

La réforme de la formation et du recrutement des enseignants en France, lancée en 2008, a suscité des débats tels que la Conférence des présidents d'université, les conférences des 
directeurs d'IUFM, des UFR de sciences et de lettres et sciences humaines ont décidé d'organiser des états généraux de la formation des enseignants. En s'appuyant sur une approche statistique, ce document propose une synthèse des différentes contributions en abordant les thèmes suivants : l'exercice du métier, les savoirs et compétences en jeu, la recherche au sein de la formation des enseignants, l'alternance, les acteurs de la formation, l'articulation mastersconcours et l'architecture des concours.

LE MENER Dominique, Enseignement scolaire : tome IV, Assemblée nationale/ Paris, novembre 2009, 80 p. téléchargées du site http://www.assemblee-nationale.fr, bibliogr.

Ce rapport pour avis défend l'importance d'une revalorisation du métier d'enseignant en France, considérée comme partie intégrante de la politique au service de la réussite des élèves. Il signale que ce métier, en pleine mutation, est notamment affecté par un niveau de formation initiale en hausse. Les mesures de revalorisation préconisées passent par une meilleure prise en compte de la performance devant les élèves et par l'accompagnement des enseignants.

POCHARD Marcel, Livre vert sur l'évolution du métier d'enseignant, janvier 2008, 271 p. téléchargées $d u$ site http://lesrapports.ladocumentationfrancaise.fr, annexes Ce rapport dresse un diagnostic de l'état de la condition enseignante en France en 2008. Puis il présente les perspectives qui s'ouvrent aux enseignants en termes d'autonomie et de responsabilités, de conditions d'exercice du métier, de recrutement, de formation et de carrière.

\section{DES COMPÉTENCES AU DÉVELOPPEMENT PROFESSIONNEL}

\section{Compétences et évaluation des enseignants}

BAILLAT Gilles dir., DE KETELE Jean-Marie dir., PAQUAY Léopold dir., et al., Évaluer pour former : outils dispositifs et acteurs, De Boeck Université/Bruxelles/ Belgique, 2008, 217 p., bibliogr. (Pédagogies en développement)

La pression de plus en plus forte qu'exercent les évaluations internationales amène les acteurs à s'interroger de manière renouvelée sur la mesure de l'efficacité de l'action de formation, sur les leviers et les régulations permettant de l'améliorer. La première partie est consacrée aux fondements des démarches d'évaluation et aborde les raisons pour lesquelles on constate aujourd'hui leur développement. La seconde partie réunit des contributions relatives aux outils d'évaluation, dans leurs rapports aux apprentissages. La troisième partie vise plus particulièrement la question de la formation des enseignants et des formateurs.

BEDIN Véronique coord., JORRO Anne coord., L'évaluation-conseil en éducation et formation: dossier, Dossiers des sciences de l'éducation (Les), 2007, $n^{\circ} 18$, $140 \mathrm{p}$.

La démarche d'évaluation-conseil sous-tend un processus dialogique permettant un travail réflexif sur les ajustements potentiels. Elle a un rôle à jouer pour favoriser la professionnalisation des enseignants ou de leurs formateurs et participe également au développement professionnel. 
FEYFANT Annie, Le contrôle du travail des enseignants contribue-t-il à la professionnalisation de leur métier?, juin 2010, 6 p. téléchargées du site http:// www.unige.ch, (Échos des colloques)

Le compte rendu de ce colloque qui s'est tenu en juin 2010 à Genève revient notamment sur l'évolution du contrôle du travail enseignant, en s'appuyant sur la modèle nord-américain. Le système finlandais est également présenté, qui repose sur l'évaluation interne à l'établissement des capacités à apprendre des élèves et sur l'auto-évaluation des enseignants. Les contributions se sont aussi intéressées au rôle du chef d'établissement.

ISORE Marlène, Teacher evaluation: current practices in OECD countries and a literature review, OECD/Paris, juillet 2009, 48 p. téléchargées du site http:// www.olis.oecd.org, bibliogr.

L'ouvrage propose un cadre conceptuel mettant en évidence les principaux éléments intervenant dans le processus d'évaluation des enseignants; il insiste sur l'importance de poser des objectifs clairs à cette évaluation. Il étudie ensuite les avantages et inconvénients des différentes approches et s'intéresse à certains points conflictuels tels que l'utilisation des résultats des élèves pour mesurer la performance des enseignants. Puis il analyse les effets de cette évaluation et les circonstances dans lesquelles elle semble plus efficace, équitable et fiable.

JORRO Anne dir., Évaluation et développement professionnel, L'Harmattan/Paris, 2007, 253 p. (Pratiques en formation)

Cet ouvrage collectif démontre la contribution de l'évaluation au développement professionnel des acteurs de l'éducation et de la formation. Il aborde notamment les référentiels, la régulation des dispositifs de formation et la place des pratiques auto et co-évaluatives dans le processus de formation et la certification. Il renvoie également aux postures des évaluateurs dans l'accompagnement des praticiens.

LAFORTUNE Louise dir., OUELLET Sylvie dir., LEBEL Christine dir., et al., Réfléchir pour évaluer des compétences professionnelles en enseignement, Presses de l'Université du Québec/Québec, 2008, 266 p., bibliogr. (Éducation intervention)

L'approche par compétences a profondément marqué le secteur éducatif dans la dernière décennie et la question de leur évaluation est clairement posée. L'ouvrage soulève des interrogations et apporte des pistes d'actions sur les modalités d'évaluation des compétences professionnelles en enseignement; dans des cours de didactique, des cours transversaux, des stages? Dans la production d'un mémoire, lors d'un bilan ou d'activités de synthèse ? Pour évaluer de telles compétences, les différents contributeurs cherchent également à définir le rôle de l'équipe professorale, les moments d'évaluation les plus pertinents, le rôle et l'utilisation de différents types de savoirs tout comme la prise en compte des compétences culturelle ou éthique.

\section{PEARSON D.D.R., Managing teacher performance in government schools,} mai 2010, 78 p. téléchargées du site http://download.audit.vic.gov.au, bibliogr., annexes Cet audit commandité par le ministère de l'Éducation australien porte sur la qualité de l'enseignement au sein des établissements publics. Celle-ci se mesure à travers la performance des enseignants et les initiatives prises par les établissements et le ministère à l'échelle centrale et régionale pour favoriser le développement professionnel des enseignants.

MONS Nathalie, "Autonomie et contrôle du travail enseignant : une enquête dans les pays européens », Revue internationale d'éducation de Sèvres, septembre 2008, $n^{\circ} 48$, p. 109-117, bibliogr.

S'appuyant sur une enquête menée par l'agence Eurydice en 2008 auprès de vingt-huit pays européens, l'article montre qu'une évolution se dessine dans la plupart des pays, qui va vers 
un accroissement du « contrôle » des enseignants et de leur travail dans quatre domaines privilégiés : la mise en place d'une multiplicité de types de "contrôles » (externes, autoévaluations, interne par le chef d'établissement ou les pairs ; ces dispositifs pouvant se superposer); une évaluation par les résultats des élèves; une évaluation qui oscille entre l'évaluation collective des équipes pédagogiques et une évaluation individuelle des enseignants ; l'absence d'incitations ou récompenses.

\section{WEISS Jacques ed., Quelle évaluation des enseignants au service de l'école?} Actes du séminaire 2007 : Leysin 6 et 7 décembre, IRDP : Institut de recherche et de documentation pédagogique/Neuchâtel/Suisse, 2008, 111 p.

Ces actes proposent un panorama des tendances actuelles de l'évaluation des enseignants dans les pays de l'OCDE. Puis une réflexion est menée sur les recommandations à faire pour l'évaluation des enseignants dans le cadre d'une réforme de l'inspection en France et sur l'évolution de ces évaluations pour dynamiser le développement professionnel et l'implication des enseignants. Enfin, une synthèse des ateliers rend compte des pratiques existantes en Suisse romande et alémanique et souligne le lien entre évaluation et démarche de formation.

\section{Compétences et développement professionnel}

CHAUVIGNÉ Christian (sous la direction de), LENOIR Yves (sous la direction de), "Les référentiels en formation. Enjeux, fondements, contenus et usages ", Recherche et formation, 2010, $n^{\circ} 64$

Les six articles de ce numéro rendent compte des différentes interprétations et applications qui peuvent être faites des référentiels en formation, plus particulièrement dans quatre pays francophones. Les approches à la fois disciplinaires, théoriques et empiriques ont été volontairement croisées.

CHUNG WEI Ruth, DARLING-HAMMOND Linda, ANDREE Alethea, et al., Professional learning in the learning profession: a status report on teacher development in the U.S. and abroad: technical report, NSDC: National Staff Development Council/Dallas/Etats-Unis, février 2009, 152 p. téléchargées du site http://www.srnleads.org, bibliogr., stat., annexes

À partir d'une recherche menée aux États-Unis et dans plusieurs pays de l'OCDE sur le développement professionnel des enseignants, ce premier rapport analyse le statut et les formes du développement professionnel - des connaissances universitaires à un système de tutorat particulièrement utile aux enseignants débutants, en passant par l'apprentissage professionnel collaboratif à l'intérieur des établissements -, les conditions de son efficacité et les instruments d'un meilleur déploiement.

GRANGEAT Michel, GRAY Peter, "Factors influencing teachers' professional competence development ", Journal of vocational education \& training, décembre 2007, vol. 59, $n^{\circ}$ 4, p. 485-501, bibliogr.

À partir d'une soixantaine d'entretiens auprès d'enseignants français, les auteurs identifient les facteurs permettant aux enseignants de s'améliorer et rendent compte de deux modèles dominants de développement des compétences professionnelles. L'échange entre enseignants et avec les partenaires de l'établissement jouent un rôle prépondérant dans la conception que les enseignants se font de leur métier. 
HOFSTETTER Rita, SCHNEUWLY Bernard, Savoirs en (trans)formation : au cour des professions de l'enseignement et de la formation, De Boeck Universitél Bruxelles/Belgique, 2009, 316 p. (Raisons éducatives)

Après une première partie consacrée aux savoirs constitutifs des professions de l'enseignement et de la formation, les auteurs s'interrogent sur la place des savoirs à enseigner et sur le rapport aux savoirs de ces professions, à travers des données empiriques et institutionnelles et des contextes historiques et culturels différents.

\section{LENOIR Yves (dir.), PASTRÉ Pierre. (dir.)., Didactique professionnelle et didactiques disciplinaires en débat : un enjeu pour la professionnalisation des enseignants, Toulouse: Octarès Éditions, 2008, 320 p.}

Le parti pris de cet ouvrage collectif, fruit d'une rencontre internationale organisée par le Centre de recherche sur l'intervention éducative (CRIE, Québec) et le Centre de recherche interuniversitaire sur la formation et la profession enseignante (CRIFPE, Québec), est que les didactiques disciplinaires, centrées sur les savoirs, et la didactique professionnelle, centrée sur l'activité, sont deux approches qui peuvent se féconder mutuellement. La deuxième partie est consacrée à la formation des enseignants.

McGRATH Simon, HONG J.-C., HORNG J.-S., et al., Developing teachers and teaching, International journal of educational development, janvier 2008, vol. $28, n^{\circ} 1$, p. 1-103, bibliogr.

Si tous les articles ont pour commun dénominateur le développement professionnel des enseignants, les situations sur le terrain sont très contrastées entre les pays. Les articles rendent compte des disparités entre les compétences demandées en formation initiale et en formation continue à Taïwan, du renouveau pédagogique dans les écoles primaires du Nigéria, de la réforme de la formation des enseignants en Turquie ou encore d'initiatives de formation continue au Vietnam.

OCDE: Organisation de coopération et de développement économiques/Paris, Creating effective teaching and learning environments: first results from TALIS, OCDE/Paris, 2009, 305 p., stat., bibliogr. Teaching and learning international survey

L'OCDE publie sa première étude sur les conditions d'enseignement et d'apprentissage, réalisée auprès de 70000 enseignants et directeurs d'établissement du premier cycle de l'enseignement secondaire de vingt-trois pays membres. Le rapport porte sur le développement professionnel des enseignants et sur leurs pratiques pédagogiques, leurs comportements et leurs perceptions. Il s'intéresse à l'évaluation des établissements et des enseignants et à l'impact de celle-ci.

\section{PERRENOUD Philippe, Dix nouvelles compétences pour enseigner : invitation} au voyage, ESF/Paris, 2008, 188 p. pédagogies références

Dans le contexte actuel de transformation du métier d'enseignant, dix grandes familles de compétences ont été retenues: l'organisation et l'animation des situations d'apprentissage ; la gestion de la progression des apprentissages; la conception et l'évolution des dispositifs de différenciation; l'implication des élèves dans leurs apprentissages et leur travail ; le travail en équipe ; la participation à la gestion de l'école ; l'information et l'implication des parents ; l'utilisation des nouvelles technologies; l'affrontement des dilemmes éthiques de la profession et la gestion de sa propre formation continue. 
TALBOT Laurent dir., BRU Marc dir., Des compétences pour enseigner : entre objets sociaux et objets de recherche, Presses Universitaires de Rennes/Rennes, 2007, 238 p., bibliogr. (Didact Education)

Même si le ministre français de l’Éducation a défini un référentiel des dix compétences requises pour l'enseignement, du point de vue de la recherche en éducation, ces compétences sont difficiles à cerner. Cet ouvrage collectif questionne la notion de compétence et réfléchit à la construction du "savoir-enseigner » en s'appuyant notamment sur les pratiques en IUFM et les référentiels utilisés, en particulier en Suisse romande.

\section{SITOGRAPHIE}

\section{Formation des enseignants}

La Commission européenne met en ligne une rubrique dédiée à la formation des enseignants. Elle y explicite les actions qu'elle mène, notamment à travers le groupe de travail qu'elle a créé sur le développement professionnel des enseignants. À travers le programme Comenius, la Commission ouvre à la formation continue des enseignants et à l'élaboration de nouvelles approches pédagogiques. Cette page regroupe également les rapports et textes juridiques de l'Union européenne sur cette question. http://ec.europa.eu/education/school-education/doc832_fr.htm

\section{IFADEM : Initiative francophone pour la formation à distance des maîtres}

Cette initiative, co-pilotée par l'Organisation internationale de la Francophonie (OIF) et l'Agence universitaire de la Francophonie (AUF) a pour mission d'améliorer les compétences des enseignants du primaire dans l'enseignement du français, en particulier les instituteurs situés en zones rurales. IFADEM a mis en œuvre un dispositif qui conjugue formation traditionnelle, utilisation des technologies de l'information et de la communication et formation à distance. Les ressources pédagogiques produites sont librement accessibles sur le site internet. IFADEM propose également de créer des sites web à vocation pédagogique. L'expérimentation concerne actuellement le Bénin, le Burundi, Haïti et Madagascar. http://www.ifadem.org/

\section{International taskforce on teacher for EFA (Education for all)}

Dans le cadre du programme d'éducation pour tous, une équipe a été formée pour coordonner et encourager l'offre éducative et la formation des enseignants. La pénurie d'enseignants est en effet estimée à dix millions de personnes pour l'enseignement primaire. Ce site, vitrine de leur action, propose des rapports et articles sur la pénurie d'enseignants et sert de plateforme pour échanger sur les bonnes pratiques. http://www.teachersforefa.unesco.org/.

\section{NéoP@ssation}

L'Institut national de recherche pédagogique a ouvert fin août 2010 sur son site web un dispositif pour la formation des enseignants, plus particulièrement destiné aux débutants. Il met en ligne des ressources réalisées à partir de travaux de recherche fondés sur l'observation du travail réel des enseignants. Les ressources, vidéo et écrites, concernent essentiellement des situations de classe, dont les situations professionnelles problématiques, commentées ou analysées par des enseignants débutants ou expérimentés et des chercheurs. http://neo.inrp.fr/.

\section{TTISSA : Initiative de l'Unesco pour la formation des enseignants en Afrique subsaharienne}

Ce projet, initié en 2006 pour dix années, a pour objectif d'améliorer les politiques nationales et de renforcer les capacités de formation des enseignants des quarante-six pays d'Afrique subsaharienne. L'Unesco assure un rôle de catalyseur et de coordonnateur; il fournit une assistance technique et méthodologique ainsi que son expertise. Les thématiques abordées sont les politiques relatives aux enseignants, le statut et les conditions de travail, la gestion des enseignants et leur développement professionnel. http://www.unesco.org/fr/ttissa/. 